\title{
Catalyst Decomposition During Olefin Metathesis Yields Isomerization-Active Ruthenium Nanoparticles ${ }^{\star \star}$
}

\author{
Carolyn S. Higman, ${ }^{\S}$ Anabel E. Lanterna, ${ }^{\S}$ M. Luisa Marin, Juan C. Scaiano* and Deryn E. Fogg*
}

\begin{abstract}
The second-generation Grubbs catalyst, $\mathrm{RuCl}_{2}\left(\mathrm{H}_{2} \mathrm{IMes}\right)\left(\mathrm{PCy}_{3}\right)(=\mathrm{CHPh})(\mathrm{GII})$, is shown to decompose during olefin metathesis to generate Ru nanoparticles. These RuNPs appear to contribute significantly to competing isomerization during metathesis. Larger, partially oxidized RuNPs are also observed in commercial GII, which exhibit modest isomerization activity. Removal of RuNPs from the precatalyst does not prevent isomerization, because new, more reactive NPs are generated by catalyst decomposition during metathesis.
\end{abstract}

Ruthenium-catalyzed olefin metathesis is a core tool in organic synthesis,${ }^{[1]}$ and an emerging protagonist in the pharmaceutical industry. ${ }^{[2]}$ Notwithstanding the importance of these advances, a number of reports cite challenges arising from competing olefin isomerization, ${ }^{[3]}$ the dominant non-metathetical side reaction encountered. ${ }^{[4]}$ Isomerization is particularly pronounced for the second-generation Grubbs catalyst GII, relative to its predecessor GI (Figure 1). ${ }^{[3]}$

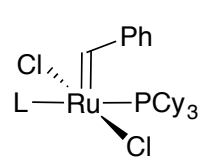

GI: $L=P C y_{3}$
GII: $L=\mathrm{H}_{2} \mathrm{Mes}$

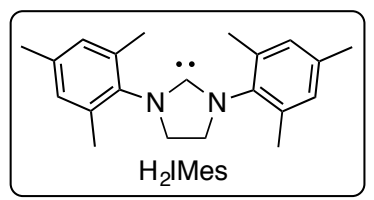

Figure 1. Grubbs catalysts $\mathrm{Gl}$ and $\mathrm{GII}$.

Tandem metathesis-isomerization or isomerizationmetathesis protocols, employed as a deliberate synthetic strategy, can enable access to targets that are otherwise challenging or inaccessible. ${ }^{[5-7]}$ More commonly, however, isomerization is an unintended, often capricious side-reaction that results in variable control over product selectivity and yields, in processes ranging from ring-closing metathesis (RCM) to cross-metathesis (CM) and metathesis polymerization. ${ }^{[2-3,8-9]}$ Ruthenium hydride complexes generated by catalyst decomposition are widely viewed as responsible. Until now, only molecular complexes have been

[*] C. S. Higman, Dr. A. E. Lanterna, Prof. J. C. Scaiano, Prof. D. E. Fogg

Center for Catalysis Research \& Innovation; Department of

Chemistry and Biomolecular Sciences

University of Ottawa

10 Marie Curie, Ottawa ON Canada K1N 6N5

E-mail: dfogg@uottawa.ca, scaiano@photo.chem.uottawa.ca

${ }^{[\star *} \quad$ Work supported by NSERC of Canada. CSH thanks NSERC for a PGS-D Scholarship. MLM was a Distinguished Visiting Professor from Universitat Politècnica de València. JCS thanks the Canada Research Chairs program; DEF thanks Magdalen College, Oxford, for a Visiting Fellowship.

§Equal contributions. Supporting information for this article is available on the WWW at http://www.angewandte.org considered as potential culprits, despite the low isomerization activity documented for leading candidates. ${ }^{[10]}$ Here we show that ruthenium nanoparticles (RuNPs) are formed by decomposition of GIl during metathesis, and that these are important, hitherto unrecognized contributors to competing olefin isomerization. It should be noted that while NP formation is common for lowcoordinate $\mathrm{Pd}$ catalysts that cycle between $\mathrm{Pd}(\mathrm{II})$ and $\mathrm{Pd}(0),{ }^{[11]}$ reports of such behaviour for well-defined ruthenium complexes operating in organic media are rare, outside hydrogenation reactions mediated by $\eta^{6}$-arene complexes of ruthenium. ${ }^{[12]}$ This is the first report of metal NP formation by decomposition of a molecular metathesis catalyst.

Olefin isomerization by RuNPs has not, to our knowledge, previously been reported. Given the activity of such species in other catalytic contexts, however, ${ }^{[13]}$ we speculated that they might function as viable isomerization catalysts. This proved to be the case. RuNPs were prepared by a range of methods (see $\mathrm{SI}),{ }^{[14-16]}$ and tested for their activity toward isomerization of estragole 1. Estragole is an important renewable allylbenzene used in metathesis reactions, ${ }^{[17]}$ which, as with its congeners, ${ }^{[9,18]}$ is readily isomerized. Figure 2 shows the isomerization activity recorded for four different Ru-containing nanostructures. All are clearly capable of inducing $\mathbf{1} \rightarrow \mathbf{2}$ isomerization. By far most active, however, were the Chaudret-Philippot NPs (Type D), prepared under rigorously anaerobic conditions, and stabilized by $\mathrm{N}$ heterocyclic carbene $(\mathrm{NHC})$ ligands. ${ }^{[14,19]}$ The dramatically higher isomerization activity of these NHC-stabilized NPs reflects the absence of oxidized surface species.

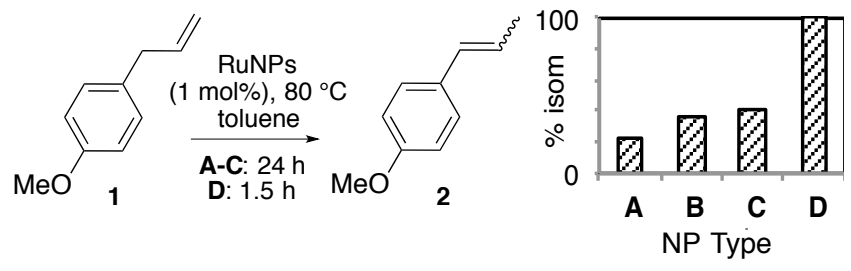

Figure 2. Isomerization promoted by RuNPs prepared by methods shown in the SI: A: RuNPs on mesoporous silica MCM-41 (Ru@MCM). B: RuNPs on crystal nanodiamonds (Ru@CND). C: RuNPs stabilized with ethylene glycol. D: RuNPs stabilized with the NHC 1,3-bis(2,6-di-isopropylphenyl)imidazole-2ylidene (IPr).

Given this evidence that RuNPs promote olefin isomerization, and prior reports that such side-reactions declined when commercial GII was chromatographed prior to use, ${ }^{[20-21]}$ we questioned whether RuNP contaminants might be present in GII, ${ }^{[2]}$ which trigger competing isomerization during metathesis. We found that commercial GIl catalysts do indeed contain RuNPs, present as aggregates that agglomerate on isolation to an average size of $>500 \mathrm{~nm}$ (see SI). However, the isolated particles induce olefin isomerization with low efficiency, requiring $24 \mathrm{~h}$ to 
reach $45 \% 2$ under the conditions of Figure 2. This is unsurprising given their large size and partial oxidation, both of which limit the number of active surface sites.

To determine whether isomerization could be inhibited by removing the RuNPs present in the precatalyst, we generated NP. free $\mathrm{GII}$ by ultracentrifugation under $\mathrm{N}_{2}$. As illustrated in Figure 3, the purified GII effected both metathesis and isomerization of estragole 1. Thus, yields of the metathesis product 3 increased over the first hour of reaction, but then declined as $\mathbf{3}$ underwent isomerization (Figure 3a). Strikingly, the extent of isomerization was only ca. $15 \%$ less than non-purified GII (Figure 3b). Freshlydecomposed Ru species thus appear to be important contributors to isomerization, with a level of activity much higher than the RuNP impurities present in the precatalyst.

$\underset{1}{\stackrel{\mathrm{Gll}}{\longrightarrow}} \mathrm{Ar}$
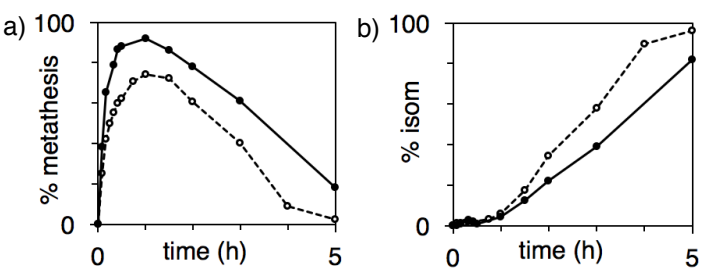

Figure 3. Performance of NP-depleted vs. NP-rich GII (solid or dashed lines, respectively), in metathesis of estragole 1. a) Formation and consumption of the self-metathesis product 3. b) Net isomerization (sum of reagent and product isomerization). See SI, S5.2.

Notable in Figure $3 \mathrm{~b}$ is the ca. 30 -min induction period that precedes the onset of isomerization. Formation of NPs over this timescale was confirmed by in situ nephelometry experiments, in which the intensity of scattered light was detected by synchronous wavelength scanning. As with conventional dynamic light scattering, increases in scattering intensity indicate NP formation. Intensity changes were monitored in the $600-700 \mathrm{~nm}$ region, to eliminate perturbation arising from absorption by the sample. The intensity of scattering increased over the first $30 \mathrm{~min}$ (see SI), a change that maps onto the induction period in isomerization. In the absence of substrate, scattering was significantly reduced.

This evidence implies that RuNPs are formed by decomposition of ruthenium species generated during metathesis We attribute the formation of nanoparticles, as opposed to molecular Ru products, to the loss of multiple ligands in the process of catalyst decomposition. Relevant in this context is the established pathway by which free $\mathrm{PCy}_{3}$, liberated from the resting-state complex GIIm (Scheme 1), attacks the methylidene ligand of the active species Ru-1. ${ }^{[23-24]}$ Elimination of the $\sigma$-alkyl ligand thus formed occurs via abstraction of a proton (most plausibly from the $\mathrm{H}_{2} \mathrm{IMes}$ ligand) and bound chloride. This process culminates in extrusion of $\left[\mathrm{MePCy}_{3}\right] \mathrm{Cl} \mathbf{A}$, a net loss of three ligands per Ru center. While isolation of the putative $\sigma$-alkyl intermediate Ru-2 is precluded by its short lifetime, we recently succeeded in trapping out such a complex in the first-generation
Grubbs system. ${ }^{[25]}$ The details of NP formation are now being probed by in situ X-ray absorption studies, but the low-coordinate $\mathrm{Ru}$ species resulting from such "ligand stripping" represent a plausible starting point.

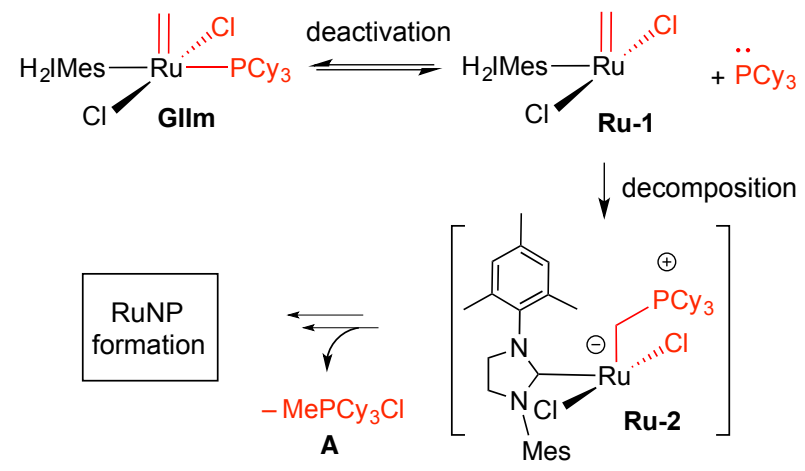

Scheme 1. Ejection of $\left[\mathrm{MePCy}_{3}\right] \mathrm{Cl}$ A from the metathesis-active species Ru-1.

Further experimental evidence for RuNP formation during metathesis comes from electron microscopy. In these experiments, styrene 4 was chosen as substrate, because the low solubility of its self-metathesis product $\mathbf{5}$ facilitates removal of organic species that otherwise occlude the micrographs. Scanning electron microscopy (SEM; Figure 4a) revealed NP-free solutions. Likewise, transmission electron microscopy (TEM) showed no NPs in analysis of multiple samples, down to the 0.2 $\mathrm{nm}$ detection level of the instrument. In contrast, abundant RuNP formation was evident following metathesis of $\mathbf{4}$, as shown in Figure 4b.

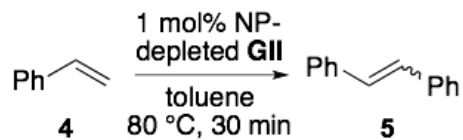

a)

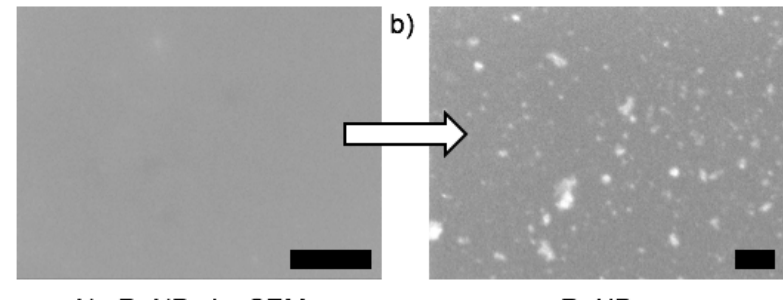

No RuNPs by SEM

RuNPs

Figure 4. Decomposition of NP-depleted GII into RuNPs during styrene metathesis. a) SEM image of GII solution prior to metathesis. b) SEM image after metathesis (COMPO mode). Scale bar: $1 \mu \mathrm{m}$. Average particle size: 100 $\pm 25 \mathrm{~nm}$.

To examine whether isomerization is promoted by RuNPs generated by catalyst decomposition during metathesis, or by molecular species formed at an earlier stage, we carried out mercury poisoning experiments. Poisoning of metal $(0)$ sites by elemental mercury is a common test for the involvement of surface-active species in catalysis. ${ }^{[26-28]}$ As shown in Figure 5, isomerization of 1 dropped by ca. $50 \%$ in the presence of $\mathrm{Hg}$. Control experiments indicated that $\mathrm{Hg}$ had negligible impact on 
the isomerization activity of common Ru hydride complexes (see $\mathrm{SI})$. Indeed, the $\mathrm{Hg}$ test may under-report the contribution of RuNPs in Figure 5, given the reported instability of the $\mathrm{Ru}-\mathrm{Hg}$ amalgam $^{[29]}$ or adsorbate. ${ }^{[26]}$

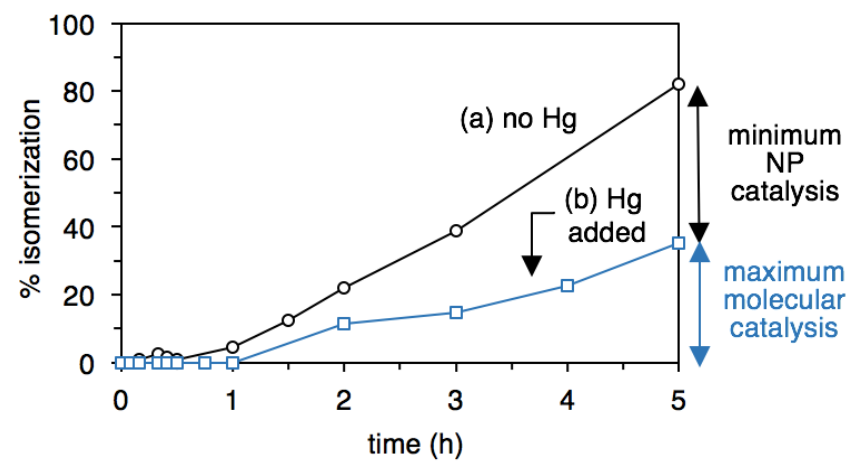

Figure 5. Impact of added $\mathrm{Hg}$ on isomerization of $\mathbf{1}$ by initially NP-depleted GII. Conditions as in Figure 3.

Sub-stoichiometric poisoning experiments (Figure 6) were carried out to further probe the involvement of RuNPs in isomerization. Such experiments are predicated on the requirement for $\geq 1$ equiv of a poisoning ligand to inhibit catalysis by molecular Ru species, in contrast with the smaller number of ligands required to inhibit NP catalysis (in which much of the initial metal charge is inaccessible in the NP core). Accordingly, we assessed the impact of $\mathrm{PMe}_{3}, \mathrm{P}(\mathrm{OMe})_{3}$ and $\mathrm{PPh}_{2} \mathrm{Me}(0.1$ equiv vs. GII) on the rate of isomerization during self-metathesis of estragole 1. These experiments were carried out at $24^{\circ} \mathrm{C}$, to maximize the poisoning effect. ${ }^{[30]}$ To compensate for the negative impact of the lower temperature on catalysis, we used a batch of estragole that showed much higher rates of isomerization. ${ }^{[31]}$ Isomerization ceased immediately on adding the phosphine / phosphite poison (Figure 6).

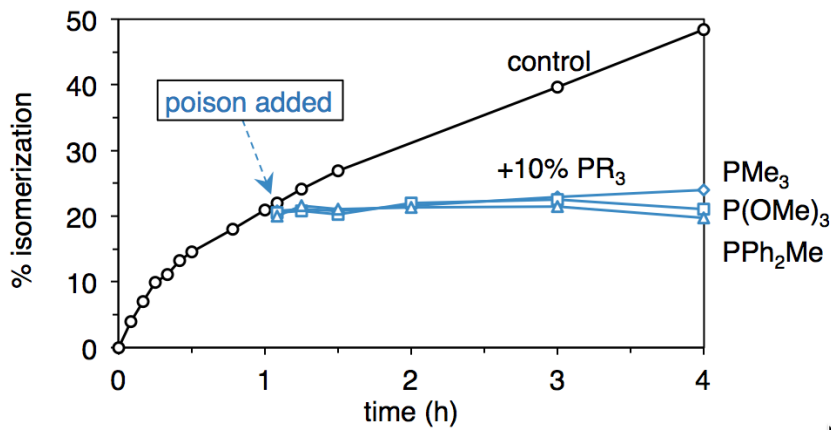

Figure 6. Sub-stoichiometric poisoning experiments: impact of adding $10 \mathrm{~mol} \%$ $\mathrm{PR}_{3}$ on the rate of isomerization during metathesis of 1 . Conditions as in Figure 3 , using NP-depleted $\mathrm{GII}$, at $24{ }^{\circ} \mathrm{C}$; poisons added at $1 \mathrm{~h}$.

The foregoing demonstrates that RuNPs can show high activity for olefin isomerization, that RuNPs are formed by catalyst decomposition during Gll-catalyzed metathesis, that $\mathrm{Hg}$ poisoning reduces isomerization, and that addition of a small proportion of a phosphine or phosphite poison, relative to the total $\mathrm{Ru}$ loading, is sufficient to completely shut down isomerization. On the basis of this cumulative picture, we propose that RuNPs formed by catalyst decomposition are important contributors to unwanted isomerization during olefin metathesis.

The context above focuses on unintended isomerization as a problem encountered during olefin metathesis. Insight into its origin, however, points toward new opportunities. The reaction conditions explored above were designed for metathesis, rather than NP formation or isomerization. Optimizing the synthesis of RuNPs, as well as the isomerization conditions, is expected to open new doors for the design of novel isomerization catalysts.

Keywords: nanoparticles $\cdot$ metathesis $\cdot$ catalyst decomposition - isomerization $\cdot$ side reactions

[1] For recent books offering comprehensive overviews, see: a) K. Grela, Ed Olefin Metathesis-Theory and Practice. Wiley, Weinheim, 2014; b) R. H. Grubbs, A. G. Wenzel, Eds. Handbook of Metathesis. 2nd ed.; Wiley$\mathrm{VCH}$, Weinheim, 2015.

[2] a) C. S. Higman, J. A. M. Lummiss, D. E. Fogg, D. E. Angew. Chem., Int Ed. 2016, 55, 3552-3565; Angew. Chem. 2016, 128, 3612-326; b) V. Farina, A. Horváth, in Handbook of Metathesis, Vol. 2 (Eds.: R. H. Grubbs, A. G. Wenzel), Wiley-VCH, Weinheim, 2015, pp. 633-658; c) K. R. Fandrick, J. Savoie, N. Y. Jinhua, J. J. Song, C. H. Senanayake, in Olefin Metathesis - Theory and Practice (Ed.: K. Grela), Wiley, Hoboken, 2014, pp. 349-366.

[3] Unintended double-bond isomerization during metathesis is discussed in recent reviews. See: a) E. Larionov, H. Li, C. Mazet, Chem. Commun. 2014, 50, 9816-9826; b) B. J. van Lierop, J. A. M. Lummiss, D. E. Fogg Ring-Closing Metathesis. In Olefin Metathesis-Theory and Practice, $\mathrm{K}$ Grela, Ed. Wiley: Hoboken, NJ, 2014; pp 85-152.

[4] B. Alcaide, P. Almendros, A. Luna, Chem. Rev. 2009, 109, 3817-3858.

[5] For recent examples of tandem catalysis strategies seeking to deliberately couple metathesis with isomerization, see: a) C. S. Higman, M. P. de Araujo, D. E. Fogg, Catal. Sci. Technol. 2016, 6, 2077-2084; b) G. E. Dobereiner, G. Erdogan, C. R. Larsen, D. B. Grotjahn, R. R. Schrock, ACS Catal. 2014, 4, 3069-3076; c) J. R. Clark, J. R. Griffiths, S. T. Diver, J. Am. Chem. Soc. 2013, 135, 3327-3330; d) B. Schmidt, S. Hauke, Org. Biomol. Chem. 2013, 11, 4194-4206; e) D. M. Ohlmann, N. Tschauder, J.-P. Stockis, K. Gooßen, M. Dierker, L. J. Gooßen, J. Am. Chem. Soc. 2012, 134, 13716-13729; f) E. Ascic, J. F. Jensen, T. E. Nielsen, Angew. Chem. Int. Ed. 2011, 50, 5188 -5191; g) T. Kobayashi, M. Arisawa, S. Shuto, Org. Biomol. Chem. 2011, 9, 1219-1224; h) C. S. Consorti, G. L. P. Aydos, J. Dupont, Chem. Comm. 2010, 46, 90589060; i) B. Schmidt, A. Biernat, Chem. Eur. J. 2008, 14, 6135-6141.

[6] Y. H. Nam, M. L. Snapper, in Handbook of Metathesis (Eds.: R. H. Grubbs, A. G. Wenzel), Wiley-VCH, Weinheim, 2015, pp. 311-380.

[7] B. Schmidt, S. Krehl, in Olefin Metathesis-Theory and Practice (Ed.: K. Grela), Wiley, Hoboken, NJ, 2014, pp. 187-232.

[8] H. Mutlu, A. N. Parvulescu, P. C. A. Bruijnincx, B. M. Weckhuysen, M. A. R. Meier, Macromolecules 2012, 45, 1866-1878.

[9] H. Bilel, N. Hamdi, F. Zagrouba, C. Fischmeister, C. Bruneau, RSC Adv. 2012, 2, 9584-9589.

[10] C. S. Higman, L. Plais, D. E. Fogg, ChemCatChem 2013, 5, 3548-3551.

[11] K. Köhler, K. Wussow, A. S. Wirth, in Palladium-Catalyzed Coupling Reactions, Wiley-VCH, Weinheim, Germany, 2013, pp. 1-30.

[12] J. G. de Vries, in Selective Nanocatalysts and Nanoscience (Eds.: A. Zecchina, S. Bordiga, E. Groppo), Wiley-VCH, Weinheim, 2011, pp. 73103.

[13] L. M. Martínez-Prieto, S. Carenco, C. H. Wu, E. Bonnefille, S. Axnanda, Z. Liu, P. F. Fazzini, K. Philippot, M. Salmeron, B. Chaudret, ACS Catal. 2014, 4, 3160-3168. 
[14] P. Lara, O. Rivada-Wheelaghan, S. Conejero, R. Poteau, K. Philippot, B Chaudret, Angew. Chem. Int. Ed. Engl. 2011, 50, 12080-12084.

[15] A. I. Carrillo, L. C. Schmidt, M. L. Marin, J. C. Scaiano, Catal. Sci. Technol. 2014, 4, 435-440.

[16] G. Viau, R. Brayner, L. Poul, N. Chakroune, E. Lacaze, F. Fiévet-Vincent, F. Fiévet, Chem. Mater. 2003, 15, 486-494.

[17] L. M. de Espinosa, M. A. R. Meier, Top. Organomet. Chem. 2012, 39, 1 44.

[18] M. Hassam, A. Taher, G. E. Arnott, I. R. Green, W. A. L. van Otterlo, Chem. Rev. 2015, 115, 5462-5569.

[19] K. Philippot, P. Lignier, B. Chaudret, Top. Organomet. Chem. 2014, 48, 319-370.

[20] A. E. Sutton, B. A. Seigal, D. F. Finnegan, M. L. Snapper, J. Am. Chem. Soc. 2002, 124, 13390-13391.

[21] K. F. W. Hekking, F. L. van Delft, F. Rutjes, Tetrahedron 2003, 59, 67516758.

[22] This hypothesis was inspired by Ananikov's discovery that PdNPs are present in commercial sources of $\mathrm{Pd}_{2}(\mathrm{dba})_{3}$. See: S. S. Zalesskiy, V. P. Ananikov, Organometallics 2012, 31, 2302-2309.

[23] S. H. Hong, A. G. Wenzel, T. T. Salguero, M. W. Day, R. H. Grubbs, J. Am. Chem. Soc. 2007, 129, 7961-7968.

[24] J. A. M. Lummiss, C. S. Higman, D. L. Fyson, R. McDonald, D. E. Fogg, Chem. Sci. 2015, 6, 6739-6746.

[25] J. A. M. Lummiss, W. L. McClennan, R. McDonald, D. E. Fogg, Organometallics 2014, 33, 6738-6741.

[26] R. H. Crabtree, Chem. Rev. 2012, 112, 1536-1554.

[27] M. Gómez, I. Favier, in Metal Nanoclusters in Catalysis and Materials Science (Eds.: B. Corain, G. Schmid, N. Toshima), Elsevier, Amsterdam, 2011, pp. 427-436.

[28] J. A. Widegren, R. G. Finke, J. Mol. Catal. A 2003, 198, 317-341.

[29] G. M. Whitesides, M. Hackett, R. L. Brainard, J. P. P. M. Lavalleye, A. F. Sowinski, A. N. Izumi, S. S. Moore, D. W. Brown, E. M. Staudt Organometallics 1985, 4, 1819-1830.

[30] J. F. Sonnenberg, R. H. Morris, Catal. Sci. Technol. 2014, 4, 3426-3438.

[31] Also notable in Fig. 6 is the absence of an induction period. These differences suggest that catalyst decomposition is accelerated by unknown impurities that are not removed by $\mathrm{Al}_{2} \mathrm{O}_{3}$ pretreatment, and which vary in proportion from batch to batch (see Introduction). For the profound, accelerating impact of additives on rates of decomposition of GII during metathesis, see refs. 24, 25 and: a) J. A. M. Lummiss, B. J. Ireland, J. M. Sommers, D. E. Fogg, ChemCatChem 2014, 6, 459-463; b) J. A. M. Lummiss, A. G. G. Botti, D. E. Fogg, Catal. Sci. Technol. 2014 $4,4210-4218$ 
